\title{
Study on multi-view calibration method in non-contact measurement
}

\author{
zhixian Zhang ${ }^{1,}$,, xujuan $\mathrm{Wu}^{2, b}$ \\ ${ }^{1}$ School of Shenyang Aerospace University, Shenyang 110135,China; \\ ${ }^{2}$ School of Shenyang Aerospace University, Shenyang 110135, China. \\ azzx_syiae@tom.com, bwxj8369@126.com
}

Keywords: binocular calibration, multi-view calibration, coordinate system transformation , translation and rotation

\begin{abstract}
This study do binocular calibration experiments on the basis of the Z. Zhang calibration method, then unify coordinate system which can determine the position of the camera with respect to the world. The whole process use image processing software halcon to perform the multi-view calibration. It is argued that the method can rapidly, accurately achieve the calibration of eight cameras in convergence mode.
\end{abstract}

\section{Introduction}

In non-contact measurement the measurement technology using image measuring technique can overcome some problems in the traditional measuring method. It can achieve high efficiency, automation, dynamic testing requirements. Therefore, applying image measuring technique to geometric measurement is one of the important development direction of geometric measurement technology in the future.

The calibration of image measurement system is the basis and prerequisite of acquiring 3D information adopting computer vision technology and is also a key link to improve 3D measurement accuracy [4]. It is to build the corresponding points relationship between space object $3 \mathrm{D}$ world coordinates and the 2D image coordinates [3]. By the relationship we can recover the depth information from dealing with collected 2D images. So camera calibration is a very important step.

Because the measurement filed is large in experiments, the monocular or binocular doesn't realize measurement requirement. In order to meet the requirement, we use eight cameras. For now, binocular calibration method is mature, for example, Tsai radial alignment constraint two-step method [1], Z. Zhang plane pattern two-step method, etc. Z. Zhang calibration method [2] [5] is applied in the experiment to get two adjacent camera's position. This part of the principle is no longer described in this paper [2] [5].

\section{Binocular vision system in convergence mode}

Binocular vision system is the simulation of human vision. It generally uses the relative position of two cameras to shoot the same object. Two or more images need to be collected. By these images compute the parallax of same points in the scenecaptured in two cameras. Then use triangulation method to get the space point coordinates.

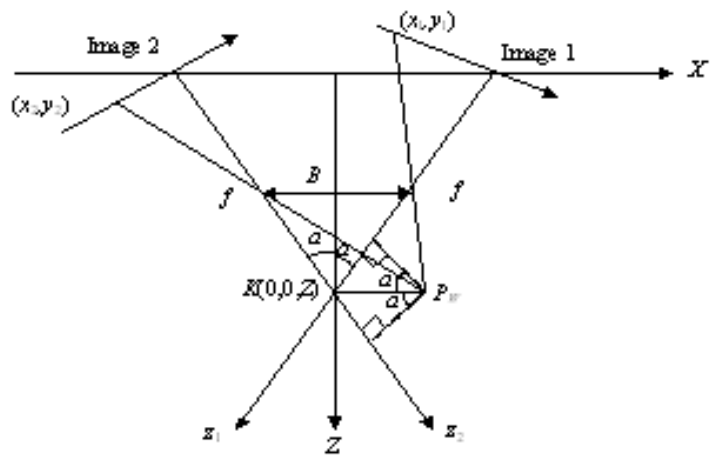

Fig. 1 Parallax figure of binocular imaging in convergence mode 
In this work, optical axis of two cameras is not parallel but in a certain angle around the centers of their projection. Namely two cameras are in convergence mod, as show in Fig. 1. The baseline is located in $\mathrm{x}-\mathrm{z}$ plane.

With a space point $\mathrm{Pw}$ corresponding to image point(x1,y1)and $(\mathrm{x} 2, \mathrm{y} 2)$ respectively known, the optical axes of two cameras intersect at a2 $\alpha$ angle to produce a point $\mathrm{K}$ or $(0,0, \mathrm{z})$ in the X-Z plane. Make two perpendicular to z1 and z2 axes respectively through the point $\mathrm{Pw}$, and we can know that the angles between the two perpendiculars and the $\mathrm{X}$ axis are both $\alpha$. By use of similar triangle calculations:

$$
\left\{\begin{array}{l}
\frac{x_{1}}{f}=\frac{X \cos \alpha}{d-X \sin \alpha} \\
\frac{x_{2}}{f}=\frac{X \cos \alpha}{d+X \sin \alpha}
\end{array}\right.
$$

Here, $d$ indicates the distance from the center of lens to node $\mathrm{k}$, which is given by:

$$
d=\frac{B}{2 \sin \alpha}-f
$$

From Eq.1 and Eq.2, we can obtain Eq.3 and Eq.4

$$
\begin{aligned}
& x_{1} r=\left(f \cos \alpha+x_{1} \sin \alpha\right) X \\
& x_{2} r=\left(f \cos \alpha+x_{2} \sin \alpha\right) X
\end{aligned}
$$

And then we can obtain Eq.5

$$
\frac{\cos \alpha}{\sin \alpha}=\frac{2 x_{1} x_{2}}{\left(x_{2}-x_{1}\right) f}
$$

From the triangle formed by the camera coordinate system axis and the world coordinate system axis. Eq.6 is given

$$
Z=\frac{B \cos \alpha}{2 \sin \alpha}
$$

Combining with Eq.5 and Eq.6, it follows that depth filed $\mathrm{Z}$ is given by

$$
Z=\frac{x_{1} x_{2} B}{\left(x_{2}-x_{1}\right) f}=-x_{1} x_{2} \frac{B}{D f}
$$

Visibly, in the case of cameras in the convergence mode, depth information has more to do with parallax and x1.x2. By the above part of the analysis, for any point coordinates in a camera imaging plane can obtain the coordinates of the point with the aid of camera imaging model as long as there are coordinates of the matching point in the other imaging plane, and then measure the geometry size of space object, restore surface topography of the object.

\section{Multi-view calibration}

From the binocular calibration, we can get each camera's internal parameters and relative position between two adjacent cameras. 


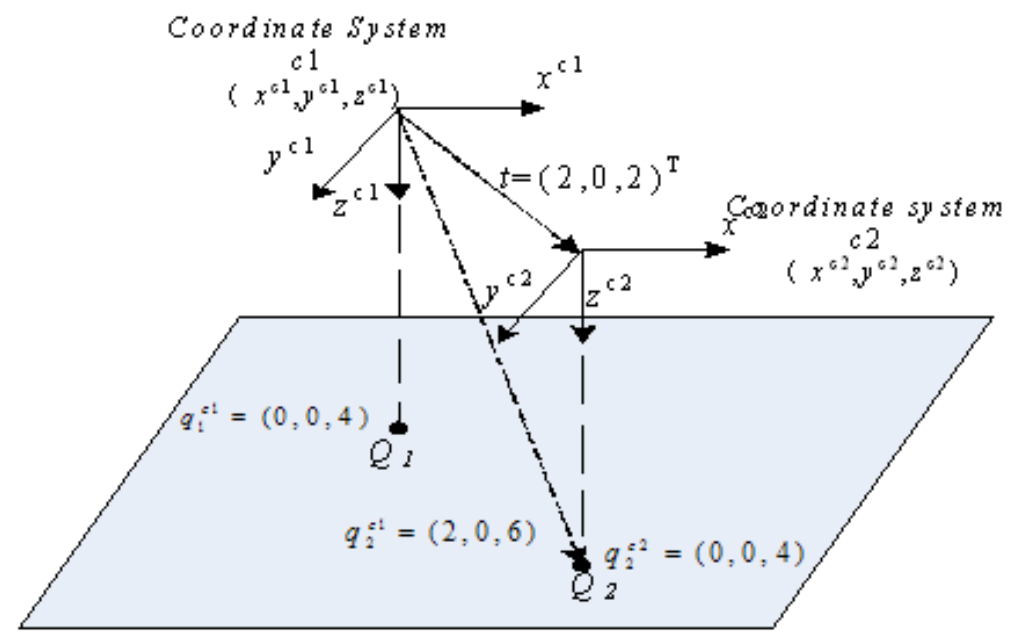

Fig. 2 the translation of coordinate system

In order to get 3D coordinate of spatial points, what we need is each camera's relationship with the world coordinate system. The key problem can be solved by the rotation and translation of the coordinate system .Fig. 2 is an instance of coordinate translation, ${ }^{c 1}$ indicates the coordinates of Q2 points in the coordinate system c1. In the same way, $q_{1}^{c 1}$ indicates the coordinates of Q1pointsin the coordinate system c1. This is depicted in Fig. 2. The coordinate systemc1, together with the point Q1, is translated by the vector t, resulting in the coordinate system c 2 and the point Q2.The points Q1 and Q2 have the same coordinates relative to their local coordinates system, i.e.

$$
q_{1}^{c 1}=q_{2}^{c 2}
$$

If coordinate systems are only translated relative to each other, coordinates can be transformed very easily between them by adding the translation vector:

$$
q_{2}^{c 1}=q_{2}^{c 2}+t^{c 1}=q_{2}^{c 2}+o_{c 2}^{c 1}
$$

In fact, this equation has been indicated vividly in Fig. 2.

It not only has the relative location relationship but also relative position relation between coordinate system and other coordinate system. If the coordinate system rotates, the position will change. Fig. 3 and Fig. 4 are instances that coordinate system c1rotate successively by y axis and $\mathrm{z}$ axis.
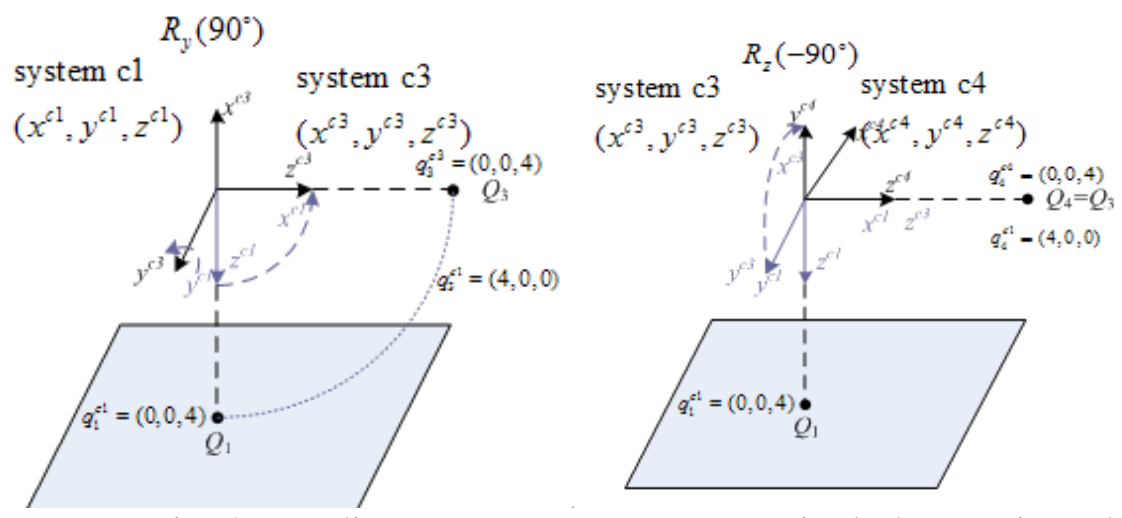

Fig. 3 coordinate system rotate successively by y axis and $\mathrm{z}$ axis

Just like the position of a coordinate system can be expressed directly by the translation vector, the orientation is contained in the rotation matrix. The columns of the rotation matrix correspond to the axis vectors of the rotated coordinate system in coordinates of the original one:

$$
R=\left[\begin{array}{lll}
x_{c 3}^{c 1} & y_{c 3}^{c 1} & z_{c 3}^{c 1}
\end{array}\right]
$$

For example, the axis vectors of the coordinate system c3 in Fig. 3 can be determined from the corresponding rotation matrix Ry (90) as shown in the following Eq: 


$$
\begin{aligned}
R_{y}\left(90^{\circ}\right) & =\left[\begin{array}{ccc}
\cos \left(90^{\circ}\right) & 0 & \sin \left(90^{\circ}\right) \\
0 & 1 & 0 \\
-\sin \left(90^{\circ}\right) & 0 & \cos \left(90^{\circ}\right)
\end{array}\right]=\left[\begin{array}{ccc}
0 & 0 & 1 \\
0 & 1 & 0 \\
-1 & 0 & 0
\end{array}\right] \\
\Rightarrow x_{c 3}^{c 1}=(0,0,-1) & y_{c 3}^{c 1}=(0,1,0) \quad z_{c 3}^{c 1}=(1,0,0)
\end{aligned}
$$

Like in the case of translation, to transform point coordinates from a rotated coordinate system c3 into the original coordinate system c1, you apply the same transformation to the points that is applied to the coordinate system c3, i.e. You multiply the point coordinates with the rotation matrix used to rotate the coordinate system c1 into c3:

$$
q_{3}^{c 1}=R_{c 3}^{c 1} \sqsubset q_{3}^{c 3}
$$

To transform point coordinates from a rotated two times coordinate system c4 into the original coordinate system c1, you need to multiply the point coordinates with the rotation matrix used to rotate the coordinate system $\mathrm{c} 1$ into $\mathrm{c} 4$ :

$$
q_{4}^{c 1}=R_{c 3}^{c 1} \square R_{c 4}^{c 3} \sqcap q_{4}^{c 4}=R_{c 4}^{c 1} \sqcap q_{4}^{c 4}
$$

Grasping the transformation of coordinate translation and rotation, we can easily unify the camera coordinate system to world coordinate system, so that we achieve multi-view calibration work.

\section{Experimentation and analysis}

In the experiment, we selected eight industrial cameras produced by German AVT company. The camera's resolution is $2752 \times 2200$ and its model is GT2750. At the same time, we choose LM12HC prime lens produced by Japanese company KOWA and its focal length is $12.5 \mathrm{~mm}$. By image acquisition software StreamPix, we got fifteen group images from different directions in the view of cameras. One group pictures are shown in Fig.4.
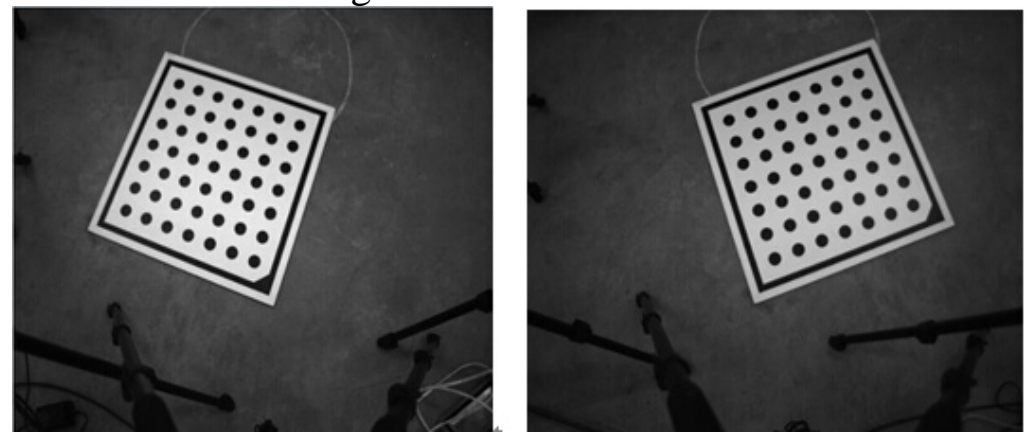

Fig. 4 image collected

According to the theory of Z. Zhang binocular calibration method, we used halcon for processing. The results are shown in Table 1 and Table 2.

Table 1 the internal experiment results of the binocular calibration

\begin{tabular}{c|c|l|c|c|c|c}
\hline & $f(\mathrm{~m})$ & $s_{x}(\mathrm{~m})$ & $s_{y}(\mathrm{~m})$ & $\begin{array}{c}c_{x} \\
(\text { pixel) }\end{array}$ & $\begin{array}{c}c_{y} \\
(\text { pixel })\end{array}$ & $k$ \\
\hline $\begin{array}{c}\text { Theinitia } \\
\text { l value }\end{array}$ & 0.0125 & $4.54 \mathrm{e}-006$ & $\begin{array}{c}4.54 \mathrm{e}-00 \\
6\end{array}$ & 1376 & 1100 & 0 \\
\hline $\begin{array}{c}\text { Camera } \\
1\end{array}$ & 0.01281 & $4.53847 \mathrm{e}-006$ & $\begin{array}{c}4.54 \mathrm{e}-00 \\
6\end{array}$ & 1378.71 & 1090.26 & $-4.59563 \mathrm{e}+6$ \\
$\begin{array}{c}\text { Camera } \\
2\end{array}$ & 0.01282 & $4.54022 \mathrm{e}-006$ & $\begin{array}{c}4.54 \mathrm{e}-00 \\
6\end{array}$ & 1394.3 & 1085.27 & $-9.13819 \mathrm{e}+6$ \\
$\begin{array}{c}\text { Camera } \\
3\end{array}$ & 0.01282 & $4.53912 \mathrm{e}-006$ & $\begin{array}{c}4.54 \mathrm{e}-00 \\
6\end{array}$ & 1376.62 & 1055.85 & $-2.86819 \mathrm{e}+6$ \\
Camera & 0.01282 & $4.54054 \mathrm{e}-006$ & $4.54 \mathrm{e}-00$ & 1367.99 & 1063.31 & $-8.11049 \mathrm{e}+6$ \\
\hline
\end{tabular}




\begin{tabular}{c|c|c|c|c|c|c}
\hline 4 & & 6 & & & \\
$\begin{array}{c}\text { Camera } \\
5\end{array}$ & 0.01287 & $4.5403 \mathrm{e}-006$ & $\begin{array}{c}4.54 \mathrm{e}-00 \\
6\end{array}$ & 1368.3 & 1103.57 & $-3.04711 \mathrm{e}+6$ \\
$\begin{array}{c}\text { Camera } \\
6\end{array}$ & 0.01282 & $4.54033 \mathrm{e}-006$ & $\begin{array}{c}4.54 \mathrm{e}-00 \\
6\end{array}$ & 1349.46 & 1056.47 & $-6.41708 \mathrm{e}+6$ \\
$\begin{array}{c}6 \\
\text { Camera } \\
7\end{array}$ & 0.01278 & $4.54127 \mathrm{e}-006$ & $\begin{array}{c}4.54 \mathrm{e}-00 \\
6\end{array}$ & 1365.05 & 1071.67 & $-3.71099 \mathrm{e}+6$ \\
$\begin{array}{c}7 \\
8\end{array}$ & 0.01283 & $4.54062 \mathrm{e}-006$ & $\begin{array}{c}4.54 \mathrm{e}-00 \\
6\end{array}$ & 1406.2 & 089.21 & $-3.00793 \mathrm{e}+6$ \\
\hline
\end{tabular}

Table 2 relative position of the binocular calibration

\begin{tabular}{c|c|c|c|c|c|c}
\hline $\begin{array}{c}\text { relative } \\
\text { position }\end{array}$ & \multicolumn{3}{|c|}{ Translation vector (m) } & \multicolumn{3}{|c}{ Rotation vector (m) } \\
\hline 1、2 & -0.64571 & -0.22269 & 0.173708 & 350.13 & 22.8962 & 44.8746 \\
2、3 & -0.79528 & -0.23302 & 0.158131 & 355.704 & 23.115 & 38.6028 \\
3、4 & -0.58621 & -0.20648 & 0.164599 & 353.574 & 21.6964 & 35.6382 \\
4、5 & -0.71697 & -0.25793 & 0.190096 & 349.492 & 22.2737 & 41.2627 \\
5、6 & -0.60322 & -0.18957 & 0.096172 & 357.661 & 20.9733 & 38.5994 \\
6、7 & -0.72371 & -0.14519 & 0.13347 & 354.006 & 24.1018 & 35.3764 \\
7、8 & -0.74057 & -0.27082 & 0.189398 & 350.484 & 23.4722 & 34.4733 \\
8、1 & -0.7191 & -0.39828 & 0.241143 & 348.113 & 22.8389 & 50.4765 \\
\hline
\end{tabular}

After the binocular calibration, we choose a group images that the calibration plate was in the center of the field, made the center of the calibration plate as the center of the world coordinate. By means of translation and rotation, we got the position relationship between eight cameras and the world coordinate. Specific parameters got are indicated in the table 6.

Table 3 eight camera positions relationship with the world coordinate

\begin{tabular}{c|c|c|c|c|c|c}
\hline $\begin{array}{c}\text { relative } \\
\text { position }\end{array}$ & \multicolumn{3}{|c|}{ Translation vector (m) } & \multicolumn{3}{c}{ Rotation vector () } \\
\hline Camera 1 & 0.276964 & -0.958095 & 1.28948 & 211.291 & 349.353 & 346.607 \\
Camera 2 & -0.424074 & -0.876372 & 1.28561 & 207.601 & 13.9452 & 29.5671 \\
Camera 3 & -0.94685 & -0.21325 & 1.30395 & 190.276 & 31.4023 & 74.0782 \\
Camera 4 & -0.828566 & 0.417851 & 1.29421 & 164.352 & 28.7283 & 121.662 \\
Camera 5 & -0.215403 & 0.908501 & 1.283 & 150.681 & 7.85185 & 165.229 \\
Camera 6 & 0.423923 & 0.87786 & 1.28967 & 147.482 & 346.895 & 203.245 \\
Camera 7 & 0.984758 & 0.378911 & 1.28566 & 164.741 & 327.841 & 244.154 \\
Camera 8 & 0.951595 & -0.431327 & 1.29841 & 194.413 & 329.139 & 292.409 \\
\hline
\end{tabular}

The calibration program need 150s to perform except image acquisition, so the time needed equally is 19.75 s to get the relationship of camera coordinate system and world coordinate system. After we measure the distance of the adjacent camera and each camera to world coordinate system center, we know the calibration error is about $2 \mathrm{~mm}$. The experimental result fully demonstrates that this multi-view calibration method is effective.

\section{Summary}

In this paper, we succeed in calibrating eight cameras but calibration precision is needed to improve. Under the condition of large measurement, more cameras may be needed to meet the requirements, therefore this calibration method can be widely used. In the next work, we will continue to improve the calibration precision and apply it to measurement. 


\section{References}

[1]Tsai R Y. An efficient and accurate camera calibration technique for 3D machine vision[C].In: Proceedings of IEEE Conference on Computer Vision and Pattern Recongnition, 1986: 364 374.

[2]Z. Zhang. A flexible new technique for camera calibration[J]. IEEE Transaction son Pattern Analysis and Machine Intelligence, 2000, 22(11): 1330-1334.

[3] Guangjun Zhang. Machine Vision [M]. Beijing: Science Press, 2005

[4]Carsten Steger, Markus Ulrich, Christian Wiedemann, Machine vision algorithms and applications[M], Beijing, Tsinghua University Press,2008

[5] Zhang Y. Zhang Z. Zhang J. Camera calibration technique with planar scenes [C]// Machine Vision Applications in Industrial Inspection XI. Santa Clara, CA, USA: SPIE, 2003: 291-296. 